Available online @ https://jiem.jnnce.ac.in https:www.doi.org/10.37314/JJEM.2021.050103 Indexed in International Scientific Indiexing (ISI) Impact factor: 1.025 for 2018-19 Published on: 30 September 2021

\title{
Blockchain Technology in Healthcare
}

\author{
Pavan N Kunchur ${ }^{1}$, Prasad Pujar ${ }^{2}$ Khasgatesh Hiremath ${ }^{3}$, Amodh Budnimath ${ }^{4}$, \\ Sadhana Bangarashetti ${ }^{5}$, Vidyadheesh Pandurangi ${ }^{6}$ \\ 1, 2,3,4, 6 Department of Computer Science and Engineering, \\ Gogte Institute of Technology, Belagavi, Karnataka, India \\ ${ }^{5}$ Department of Information Science and Engineering, \\ Basaveshwara Engineering College, Bagalkot \\ 1pnkunchur@git.edu, 20mpujar@git.edu, ${ }^{3}$ cosbahubali1997@gmail.com, ${ }^{4}$ amodhbudnimath@gmail.com \\ 5sadhanapb@gmail.com, 6vjpandurangi@git.edu
}

\begin{abstract}
This article describes the implementation of Blockchain Technology in the HealthCare domain, using the Ethereum framework is an important tool. This is a proof of concept application (PoC); Since, it is Blockchain applications (Dapp) are heavily dependent upon smart-contracts, the application is required to use a solidity framework, ganache, Web3.js for Front-end development. This application shows how patient data can be retrieved and stored in the Blockchain cloud. The designed system also helps the doctors to directly connect to their patients. The opinion of experts can be made available to the patients.
\end{abstract}

Keywords: Ethereum framework, Smart-contracts, hashcode, Blockchain, Ganache

\section{Introduction}

The confidentiality of today's global data becomes a myth, thanks to traditional archival knowledge, processing, and third-party data consent. The healthcare sector is one of the areas where data privacy is often overlooked. There are cases where patient data is misused The Health Insurance and Accountability Management Act (HIPAA) states a standard for the protection of sensitive patient data. Companies dealing with protected health information (PHI) must have the physical, network, and operational measures in place and ensure that they comply with HIPAA compliance. Associated organizations (anyone providing treatment, payment, patient sharing, and health care services) and business partners (anyone who can access patient information and provide treatment, payment, or performance) must meet HIPAA compliance. must provide computer services such as payments, doctor's distribution, Electronic Health Records (ERH) over which the patient has control more than his data. Keeping all of this in mind, medical data is safe and secure by the well-known Blockchain application health application currently. Blockchain can maintain an unbreakable, low-quality, and transparent log for all patient data making Blockchain technology much better than standard applications. Blockchain technology creates unique opportunities to reduce complexity, allow for seamless interaction, and create secure and consistent data. HHS is ready to follow up on this rapidly evolving field to identify trends and sensible areas where government support may be needed for the technology to achieve its full potential in health care. To shape the future of the 
Blockchain, HHS should consider mapping and integrating the Blockchain system, establishing a Blockchain framework for first-time users, and supporting negotiation and acquisition alliances.

\section{Literature survey}

Ten years ago Satoshi Nakamoto ${ }^{[5]}$, a man or group leader who coined the name bitcoin and explained how the Blockchain works. The first low-level ledger was designed and used by Satoshi Nakamoto. This leads to the massive development of a separate application on different domains. In 2008, First an engineer or team of developers working under Satoshi Nakamoto who published a white paper on Model on Blockchain. In fact, the History of the Blockchain concept goes back to 1991 as a series of secretly protected blocks was first described by Stuart Haber and W Scott Stornetta. As evolving financial technology advances in mid-1998 Computer scientist, Nick Szabo works on 'Bit gold' as a partial digital currency. However, in 2009 Nakamoto used the first Blockchain as a permanent public transaction log using bitcoin. In the business world trusting on third-party applications is always less trustworthy and fear of leaking sensitive data to dangerous people. After the massive development of the Blockchain and its structures, many industries such as Textile, supply chain management, ${ }^{[1]} \mathrm{CRM}$, ERP have used Blockchain to build reliable customer applications. Blockchain can bring transaction clarity to a new level, but at the moment the acceptance of education and Blockchain technology management is limited by our understanding. To address this issue, this study uses the Integrated Theory of Adoption and Use of Technology (UTAUT) and the concept of adoption of new technological devices as the basic framework for tracking procurement.

\section{Blockchain}

Blockchain is a Peer-to-peer distributed ledger, unlike a traditional centralized system. It contains three important components mainly -
1. A distributed networking

2. Sharable ledger

3. Digital transaction(immutable)

\subsection{Distributed networking}

Distributed P2P shared architecture with block nodes containing participants in the blockchain network. Each participant in the network maintains the same copy of the Blockchain and contributes to a collaborative authentication process, verifying digital signatures of digital transactions taking place on the Blockchain network.

\subsection{Sharable Ledger}

Participants in the distributed Blockchain network record digital transactions into systematic shared ledger. To add transactions, participants in the network run algorithms to verify and evaluate. These are called "Minors" which are complicated underlying algorithms in Blockchain network. If majority of participants in approves these transactions are added to shared ledger. If any changes in shared ledger will reflect in all copies of the ledger in the distributed network. After a transaction is added, it is immutable and cannot be changed or removed. Since all members in the network have a complete copy of the Blockchain, no single member has the power to tamper or alter data. Sharable ledgers in a Blockchain network is shown in figure 1.

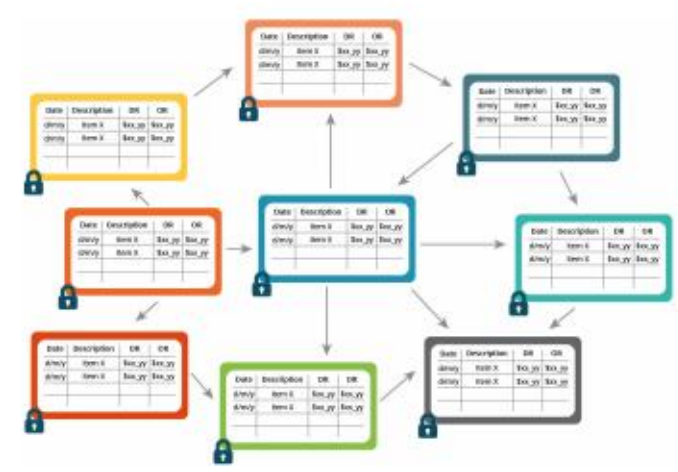

Figure 1: Sharable ledgers in a Blockchain network 


\subsection{Digital Transaction}

Any type of data or digital assets can be stored in a blockchain, using the Network means any type of data is stored in a transaction, encrypted and digitally signed with authenticity and security. Transactions are designed as blocks and each block has a previous block hash in the blockchain (figure 2 ). The blocks are placed in sequence, in chronological order.

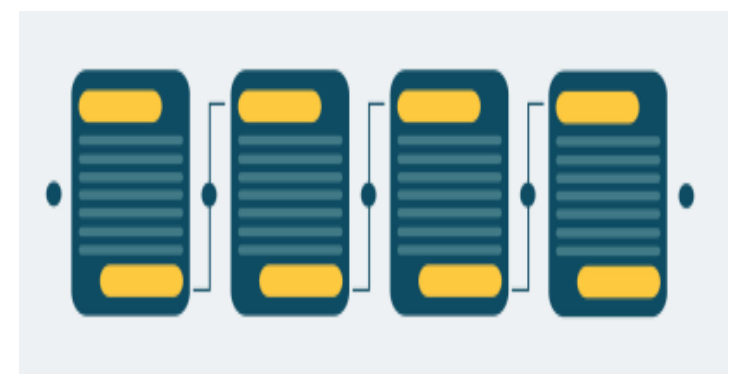

Figure 2: Blocks in a Blockchain network

\section{Methodology}

\subsection{Purpose}

The primary purpose of this $\mathrm{PoC}$ is to prove that Blockchain can be implemented in the Ethereum Framework for Healthcare. The aim is to develop an application which can be used by patient, hospitals, and doctors to securely store, transfer, and retrieve medical records between the stakeholders.

\subsection{Scope}

This project will consist of creating a prototype solution to prove the Proof of Concept of using the Ethereum Framework to implement Blockchain.

\subsection{Definitions}

\subsubsection{Blockchain}

A blockchain is, in simple terms, a series of timeless data stamps owned by a non-commercial computer network. Each of the data blocks (e.g. Block) is secured and tied to each other using cryptographic terms (i.e. chain).

\subsubsection{Ethereum}

Ethereum is a global, open-source platform for decentralized applications. On Ethereum, you can write code that controls digital value, runs exactly as programmed, and is accessible anywhere in the world.

\subsubsection{Smart-Contracts}

Smart contracts are where all the business logic of the application lives. This is where we code the decentralized portion of the app. Smart contracts are in charge of reading and writing data to the blockchain, as well as executing business logic.

\subsubsection{Solidity}

Solidity is an object-oriented, high-level language for implementing smart contracts. Smart contracts are programs that govern the behavior of accounts within the Ethereum state. Solidity is a statically typed scripting language that does the process of verifying and enforcing the constraints at compile-time as opposed to run-time.

\subsubsection{Truffle}

Truffle is a Development Environment, Testing Framework, and Asset pipeline for Ethereum Blockchains.

This PoC is proof that what exactly the system is. It also explicates how Blockchain has the potential to revolutionize the health care industry. Also how this the prototype can help and increase a lot of communication among various potential players in the healthcare industry facilitating a smooth and secure access to data pertaining only to their needs.

\subsection{Product Perspective}

1. This application will bring together all the stakeholders in the healthcare industry. 
2. This application will bring together all the stakeholders in the healthcare industry.

3. All the transaction logs will be saved in the decentralized network itself.

\subsection{Product functions}

1. The product will be designed to register new patients and doctors.

2. The patient will be the only party to have control over his/her data/health records.

3. When a new doctor registers, his/her data will be directly stored in the Blockchain and can be viewed by the hospital.

4. The hospital will be responsible to assign a doctor to each patient.

5. Only when the patient permits to access his/her data, the doctor can view the patient records.

6. The decentralized network will enable users from different parts of the globe to leverage the application to their advantage.

7. Every activity that will take place via the application will be logged for future reference to hold every party accountable for their actions.

\subsection{Assumptions and dependencies}

1. For a user to use the application on a computer, he/she would require a computer with a browser.

2. Each user will need to have a stable Internet connection to use the product.

3. Each user will need to have a stable Internet connection to use the product.

\subsubsection{Steps to build this decentralized application:}

We need to install the following dependencies

\section{a. NPM and Node.js}

1. Go to https://nodejs.org

2. Download the latest version of node.js and install it.

3. To check if you have Node.js installed, run this command in your terminal:
$\$$ node- $\mathrm{V}$
(v12.3.1)
$\$ n p m-V$
(v6.9.0)

(Note: npm is distributed with Node.js- which means that when you download Node.js, you automatically get $\mathrm{npm}$ installed on your computer.)

\section{b. Truffle}

Run this command in your terminal:

$\$$ npm install $-\mathrm{g}$ truffle

To check if you have Truffle installed, run this command:

$$
\$ \text { truffle }-\mathrm{v} \quad(\mathrm{v} 5.0 .20)
$$

\section{c. Ganache}

1. Go to http://truffleframework.com/ganache/

2. Download and install the latest version of ganache.

\section{d. Web3}

1. Open a command prompt.

2. Run this command in your terminal:

\$ npm install web3

3. To check if you have web3 installed, run this command:

\$ npm view web3 version

\subsection{Implementation}




\subsubsection{Development stages}

1. Write the required smart contract in https://remix.ethereum.org/

2. Use remixes IDE to test the smart contracts. (Note: We use remix IDE because it is easy to compile and debug a smart contract and we can even deploy the contracts for testing.)

3. Open a command prompt and create a directory for the application.

4. Run this command to download the package:

$\$$ truffle unbox pet-shop

(Note: we can also start from scratch by using the command (\$ truffle init) but pet-shop is ready to use package which contains all the dependencies and comes with a demo contract for Dapps)

5. Copy the contract from the remix and paste it into a new .sol file in the directory named contracts which are found in the pet-shop package.

6. Clear the app.js already present in the truffle package and write your web3.js interface to call and use the smart contracts.

7. Write the front end interface as required.

Figure 3 shows the transaction ID for patients transaction.

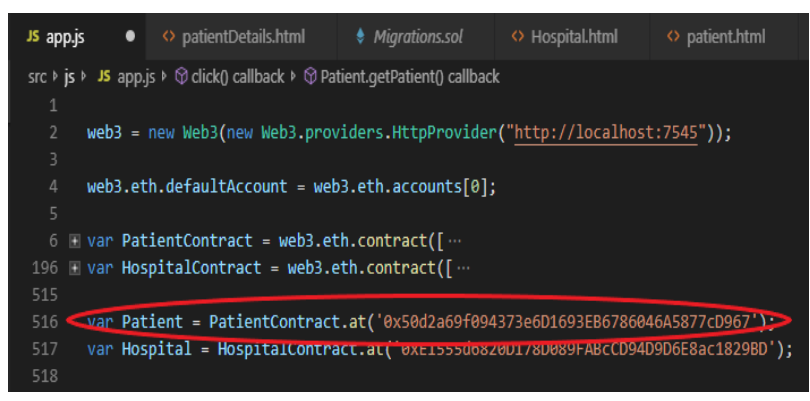

Figure 3: Transaction ID for patients transaction

\section{Procedure}

The above-mentioned dependencies must implement this PoC.

a. Open the directory containing the code in any code editor.

b. The directory structure should look like this (figure 4).

c. Run ganache from start and click on Quick start. It should look like this (figure 5).

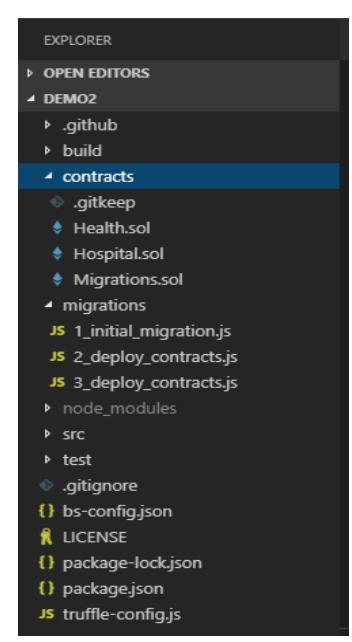

Figure 4: Project directory

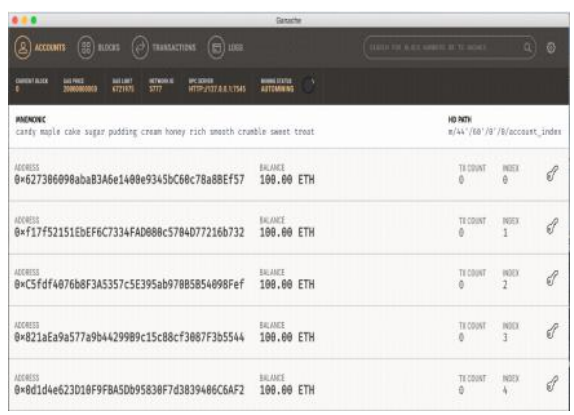

Figure 5: Ganache Interface

d. Open a command prompt and navigate to the application directory.

e. Run the following command in the prompt: $\$$ truffle migrate -reset

The smart contracts will be compiled and deployed on the ganache network. You will see the details of three deployed contracts i.e. Migrations, Hospital and Health. 
f. Copy the contract address from the Hospital contract (figure 6).

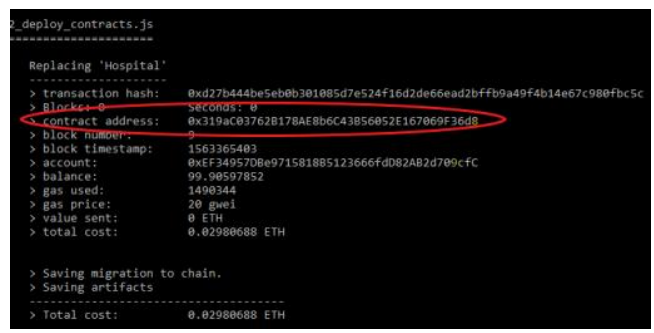

Figure 6: Transactions of the Hospital

g. Paste this address at these locations in the app.js file (figure 7).

var Hospital = hospitalcontract.at(' here')

Patient.request_doc('here',\$("\#P_reqpid").val());

Assign patients transaction to doctor (figure 8)

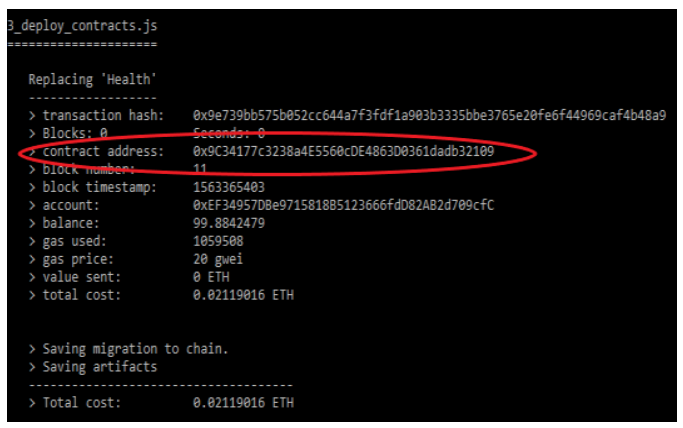

Figure 7: Contract address

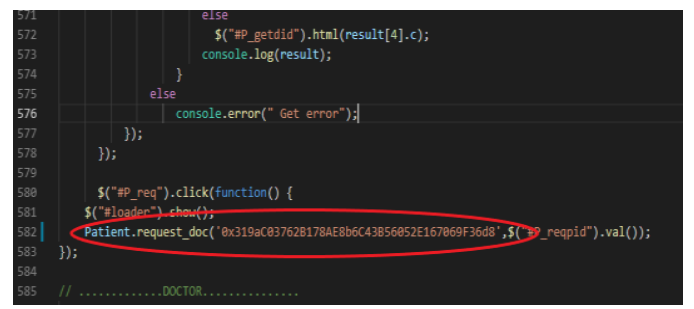

Figure 8: Assigning Patients transaction to Doctor.

(Note: Use the line numbers shown in the images as reference)Copy the contract address from the Health contract.

Paste this address at these locations in app.js file var Patient $=$ PatientContract.at('here');

Hospital.view_patient('here',\$("\#D_Pdid").val(),\$ ("\#D_Ppid").val(),function(error, result)

Hospital.setPid_and_Did('here',\$("\#H_assign_pid"). val(), \$("\#H_assign_did").val());
Hospitals transaction ID allotment is shown in figure 9.

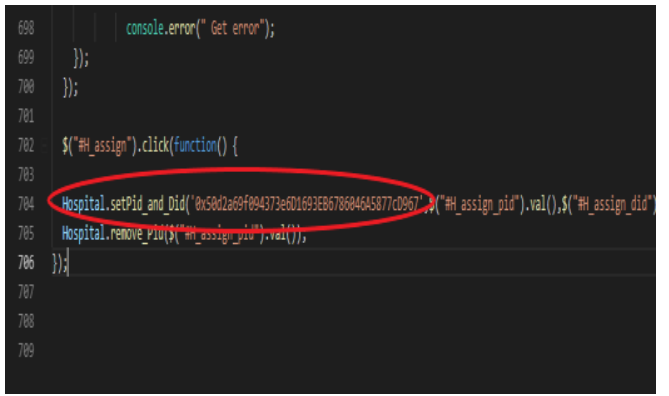

Figure 9: Hospitals Transaction ID allotment. (Note: Use the line numbers shown in the images as reference)

\section{Results and Discussion}

Above mentioned section completes the whole implementation of the Ethereum framework. Now let us go through the actual user interface of the PoC

Above mentioned section completes the whole implementation of the Ethereum framework. Now let us go through the actual user interface of the PoC

1. Run this command in the command prompt:

\$ npm run dev

Now the index page will be visible on the browser window (figure 10).

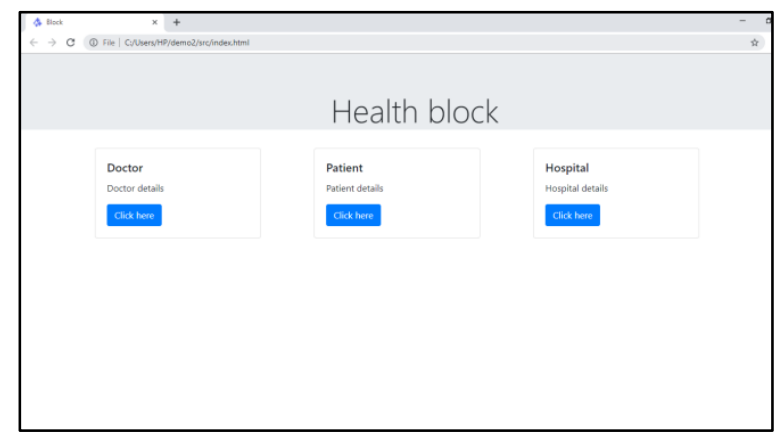

Figure 10: Index page of Application

2. Fill in all the details and click on Submit Details, and you will get the block hash (figure 11). 


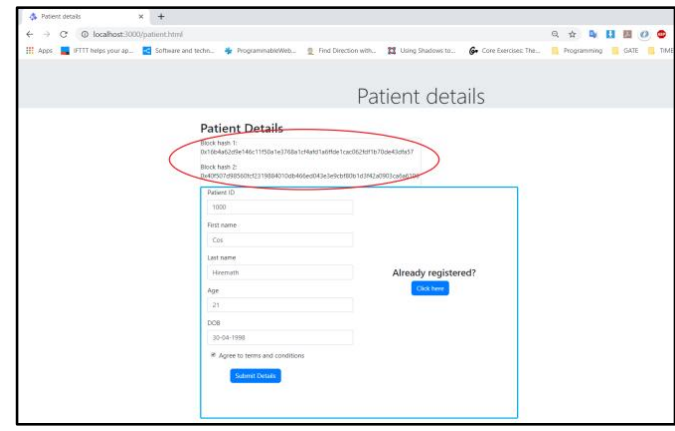

Figure 11: Patient Details

3. Once you register as a patient, you can click on the button already registered to view your data or request a hospital for a doctor to be assigned by entering your Patient Id(figure 12).

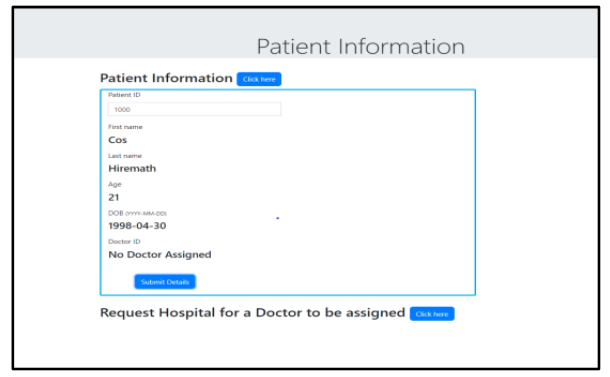

Figure 12: Patient Interface

4. For registering as a Doctor, click on Doctor details on the home page which will redirect you to the doctor's registration page.

Fill in all the details (figure13) and click on submit to upload the details to Blockchain. We will get a block hash value above.

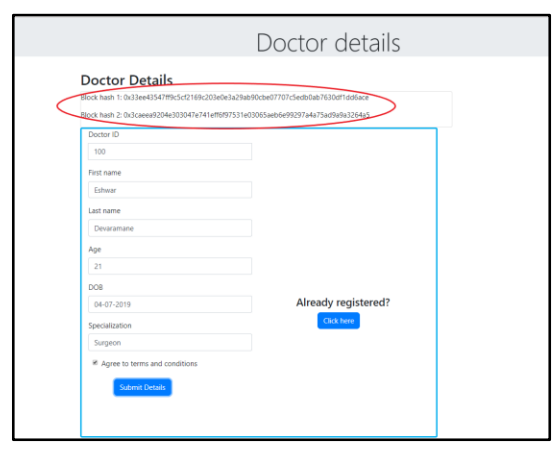

Figure 13: Doctor Details

5. Once you register as a Doctor, you can click on the button below already register to view your data or view your patient's data by entering your Doctor's and Patient's Id (figure 14).

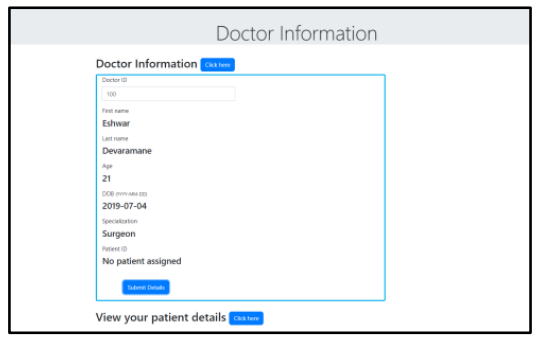

Figure 14: Doctor Information

6. On the hospital page (figure 15), you can click on the List of registered doctor's button and the List of patients requesting a doctor to view the Ids.

7. Now the hospital can assign a particular patient to a doctor by taking the above list as a reference.

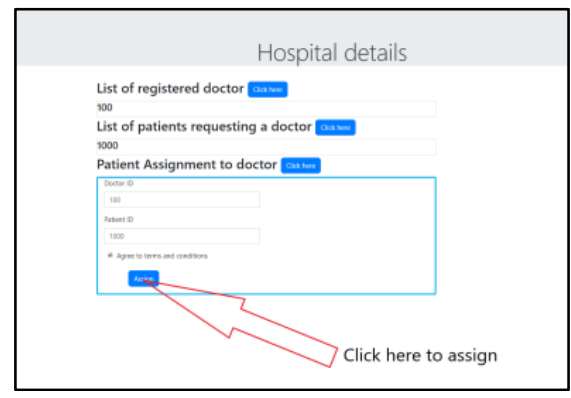

Figure 15: Hospital details

8. Now, you can go to the Patient's page (figure 16) and when you check your uploaded data, you can see the ID of the doctor who is assigned to you by the hospital.

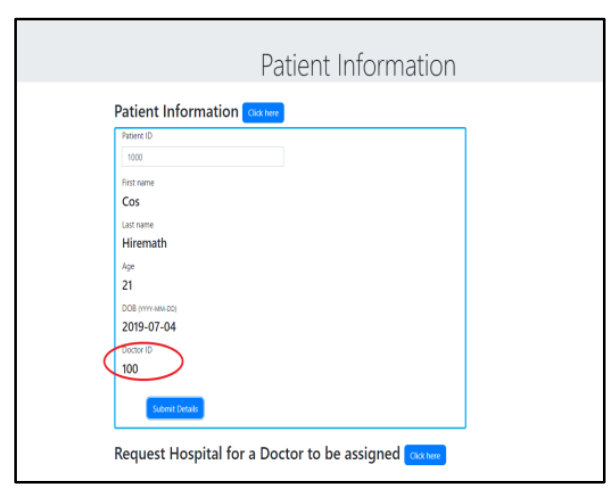

Figure 16: Patient Allocation to a Particular Doctor 
9. In the same way, you can go to Doctor's page and when you click on view data you will see the ID of the patient assigned to you.

If you click on the view your patient's details button, you can input Doctor's and Patient's ID to view your patient's data (figures 17 and 18).

(Note: If the Patient ID you have entered is not assigned to you, then you will get an alert saying that "This patient is not assigned to you")

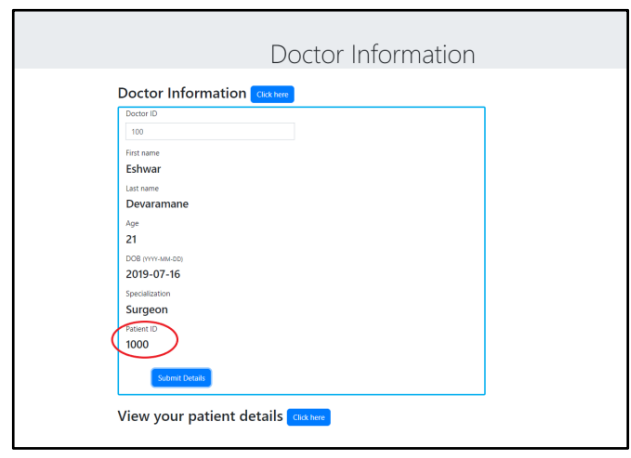

Figure 17: Patient ID through Doctor View.

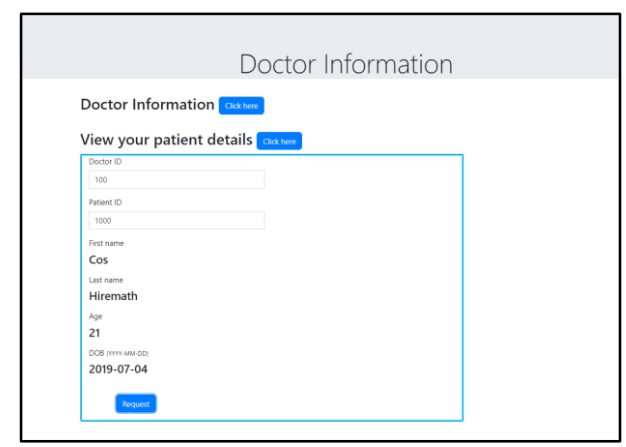

Figure 18: Doctor Information

\section{Conclusion}

A health care blockchain would expand the acquisition of health data to include data from populations of people who are currently under-served by the medical community or who do not typically participate in research. The shared data environment provided by Blockchain makes it easier to engage "hard-toreach" populations and develop results more representative of the general public. Blockchain technology offers many advantages to medical researchers, health care providers, caregivers, and individuals. Creation of a single storage location for all health data, tracking personalized data in real-time, and the security to set data access permissions at a granular level would serve research as well as personalized medicine.

\section{Future Scope}

Utilization of the proposed health blockchain described in this paper has the potential to engage millions of individuals, health care providers, health care entities, and medical researchers to share vast amounts of genetic, diet, lifestyle, environmental, and health data with guaranteed security and privacy protection. The acquisition, storage, and sharing of this data would lay a scientific foundation for the advancement of medical research and precision medicine, help identify and develop new ways to treat and prevent disease and test whether or not mobile devices engage individuals more in their health care for improved health and disease prevention. Blockchain technology definitely has a place in the health IT ecosystem. Open API's based on industry best practices are vital and essential to addressing interoperability. However, open APIs are essential but not sufficient. A shared distributed infrastructure that provides a comprehensive view of an individual's health data across a lifetime is an equally essential component of interoperable health IT systems. Blockchain technology addresses interoperability challenges, is based on open standards, provides a shared distributed view of health data, and will achieve widespread acceptance and deployment throughout all industries. The real-time availability of mobile applications and wearable sensor data from the blockchain would facilitate continuous, 24 hour-a-day monitoring of high-risk patients and drive the innovation of "smart" applications that would notify caregivers and health providers if a patient reached a critical threshold for action. Care teams could reach out to the patient and coordinate treatment options for early intervention. 


\section{References}

1. https://www2.deloitte.com/us/en/pages/publ ic-sector/articles/blockchain-opportunities-forhealth-care.html.

2. Hannah S Chen, Juliet $\mathrm{T}$ Jarrell, Kristy A Carpenter, David S Cohen, and Xudong Huang, Blockchain in Healthcare: A PatientCentered Model. Biomedical Journal of Scientific \& Technical Research, Vol. 20, No.3, August 2019, pp. 15017-15022.

3. Tiana Laurence, Blockchain for dummies, ISBN 978-1-119-55513-1, Wiley Publication, Second Edition, 2019.

4. Zyskind, Guy, and Oz Nathan. Decenterilized privacy: Using blockchain to protect personal data." Proceedings of the 2015 IEEE Security and Privacy Workshop (SPW), San Jose, California, USA. May 21-21, 2015, pp 180-184.

5. S. Nakamoto, Bitcoin: A peer-to-peer electronic cash system, White paper, October. 2008, https://bitcoin.org/bitcoin.pdf.

6. A. Azaria, A. Ekblaw, T. Vieira and A. Lippman, MedRec: Using Blockchain for Medical Data Access and Permission Management, Proceedings of the $2^{\text {nd }}$ International Conference on Open and $\mathrm{Big}$ Data (OBD), Vienna, Austria, 2016, pp. 25-30. 